\title{
Application of the 3D common-reflection-surface stack workflow in a crystalline rock environment
}

\author{
K. A. Ahmed, B. Schwarz, and D. Gajewski \\ University of Hamburg, \\ 20146 Hamburg, Germany
}

Email: khawar-ashfaq.ahmed@zmaw.de

(March 14, 2017)

Running head: 3D CRS in crystalline environment

\begin{abstract}
Seismic data from crystalline or hardrock environments usually exhibit a poor signal-tonoise $(\mathrm{S} / \mathrm{N})$ ratio due to low impedance contrasts in the subsurface. Moreover, instead of continuous reflections we observe a lot of steeply dipping events resembling parts of diffractions. The conventional seismic processing (common midpoint stack and Dip moveout) is not ideally suited for imaging such type of data. Common-reflection-surface stack processing considers more traces during the stack than common midpoint processing and the resulting image displays a better $\mathrm{S} / \mathrm{N}$ ratio. In the last decade, the common-reflection-surface stack method was established as a powerful tool to provide improved images, especially for low fold or noise contaminated data. The common-reflection-surface stack and all attributes linked to it are obtained using a coherence-based automatic data-driven optimization procedure. In this work we applied the common-reflection-surface stack workflow to 3D crystalline rock seismic data which were acquired near Schneeberg, Germany, for geothermal exploration. The common-reflection-surface stack itself provided an image of good S/N ratio. However,
\end{abstract}


for data from environments with low acoustic impedance and poor velocity information, the coherence which is automatically obtained in the optimization procedure provides an alternative way to image the subsurface. Despite the reduced resolution, for this data the coherence image provided the best results for an initial analysis. Utilized as a weight, the coherence attribute can be used to further improve the quality of the stack. By combining the benefits of a decreased noise level with the high resolution and interference properties of waveforms, we argue that these results may provide the best images in an entirely data-driven processing workflow for the Schneeberg data. 


\section{INTRODUCTION}

3D seismic imaging is a challenge for data acquired in hardrock environments. The reason for this is the small reflectivity/acoustic impedance and signal-to-noise $(\mathrm{S} / \mathrm{N})$ ratio compared to data from sedimentary basins (e.g., Milkereit et al. 2000; Malehmir et al. 2012). Contrary to data from sedimentary environments where we observe continuous events over large lateral distances, hardrock data are usually dominated by diffractions, or parts of diffraction events which leads to a criss-cross pattern and numerous conflicting dip features in the stacked sections. This challenges any kind of geological interpretation. Because of the often small lateral extent of events in hardrock data, velocity determination is difficult. Moreover, the velocity in hardrock environments is usually high and the resulting moveouts are small which provides an additional challenge in the data processing. These problems in velocity analysis influence the quality of the stacked data and may lead to an unsatisfactory image not very suitable for geological interpretation.

It was demonstrated previously that the common-reflection-surface (CRS) stacking method generates improved stack images for low fold and/or low $\mathrm{S} / \mathrm{N}$ data when compared with common midpoint stacking (e.g., Hertweck et al. 2007; Baykulov et al. 2011). This observation suggests to apply the CRS method to hardrock data. The fold in CRS processing is considerably higher than the fold in CMP processing which helps to image weak events and to improve the $\mathrm{S} / \mathrm{N}$ ratio of the stacked section. The CRS method (e.g., Mann et al. 1999; Bergler et al. 2002) is an automatic entirely data-driven approach. In addition to the stack itself it provides several kinematic wavefield attributes and the corresponding coherence of the local wavefield for each sample in the stacked data volume. The wavefield attributes 
have many applications in subsequent processing like multiple suppression (e.g., Dümmong and Gajewski 2008), NIP-wave (normal incidence point wave) tomography (e.g., Duveneck 2004), diffraction processing and imaging (e.g., Dell and Gajewski 2011), pre-stack data enhancement, data interpolation and regularization (e.g., Baykulov and Gajewski 2009) just to name a few.

In a joint project with the Leibniz Institute for Applied Geophysics (LIAG), Hannover, Germany and TU Bergakademie Freiberg, Freiberg, Germany, a 3D reflection seismic experiment was conducted in the area of the city of Schneeberg, Saxony, Germany. The field work was part of a pre-site survey for a geothermal exploration project. For details on the acquisition of the reflection seismic experiment we refer to (Lüschen et al. 2015). The subsurface in this area is complex and dominated by steep faulting in the crystalline rocks. The data show a lot of scattering due to the fractured zones and hydrothermal veins. The processing of this type of seismic data is a challenge because of the above-mentioned reasons. Conventional CMP-DMO-based processing did not provide satisfactory results for the Schneeberg data. Therefore, 3D CRS processing was applied to achieve a higher $\mathrm{S} / \mathrm{N}$ ratio and to obtain an interpretable stacked volume. In contrast to CMP processing, also neighboring CMPs are considered in the CRS approach. This feature increases the number of traces in the stack. The applied workflow is entirely data-driven in an attempt to generate an image simply based on the local coherence of the recorded wavefield.

In the following section, we briefly discuss the foundations of the 3D CRS method before we introduce the area of investigation in some more detail. The application of the CRS method to 3D data is then discussed in the following sections. The data-driven automatic 
CRS approach delivers stacked sections and several volumes of wavefield attributes, which provide a first image of the subsurface. A brief discussion of the major events in the sections and some conclusions finalize the paper.

\section{BASICS OF THE 3D COMMON-REFLECTION-SURFACE STACK WORKFLOW}

The CRS stack (e.g., Mann et al. 1999) was originally developed to obtain simulated zerooffset (ZO) sections or volumes from seismic multi-coverage data. The method is based on a traveltime surface that is of second order in the midpoint $\left(\mathbf{x}_{m}\right)$ and half-offset $(\mathbf{h})$ coordinates, i.e., it is of hyperbolic shape. The CRS stacking operator at a given zero-offset sample $\left(x_{0}, t_{0}\right)$ is determined by parameters related to the coefficients of the second order travel time expansion. For each zero-offset sample to be simulated, the optimum stacking operator is found by varying these parameter values within predefined boundaries. These parameters or attributes define the shape of the traveltime surface. Performing a coherence analysis in the pre-stack domain provides the attributes with the best fit to the data. The parameters which yield the highest coherence value describe the optimum stacking operator.

\section{D common-reflection-surface stack}

In the 3D case (e.g., Bergler et al. 2002; Müller 2003), the traveltime surface is formulated in terms of the two symmetric $2 \times 2$ curvature matrices $\mathbf{M}_{n i p}$ and $\mathbf{M}_{n}$ respectively and the two-component slope vector $\mathbf{p}_{m}$, leading to an eight parameter expression:

$$
t^{2}\left(\Delta \mathbf{x}_{m}, \mathbf{h}\right)=\left(t_{0}+\mathbf{p}_{m} \Delta \mathbf{x}_{m}\right)^{2}+2 t_{0}\left(\Delta \mathbf{x}_{m}^{T} \mathbf{M}_{n} \Delta \mathbf{x}_{m}+\mathbf{h}^{T} \mathbf{M}_{n i p} \mathbf{h}\right)
$$

where $\Delta \mathbf{x}_{m}$ denotes the relative midpoint displacement. Because the CRS operator can 
be derived by a Taylor expansion of the traveltime, in midpoint and half-offset coordinates, the stacking parameter vector $\mathbf{p}_{m}$ comprises two parameters which are related to first traveltime derivatives with respect to midpoint coordinates and the stacking parameters $\mathbf{M}_{n i p}$ and $\mathbf{M}_{n}$ are related to second order traveltime derivatives with respect to midpoint and half-offset coordinates. As long as the processing stays in time, no physical interpretation of the parameters is required and they can be considered as pure fitting coefficients of an operator to best fit the data. The CRS method and the stacking parameter estimation is an entirely data-driven approach where the optimization of the operator is performed with the coherence as objective function. The coherence measure used in this work is the semblance. The stacked images generated according to this methodology are free from any interpretation. The events in the stack are based on maximum coherence of the local wavefield for the particular zero-offset sample.

For the use of the attributes in processing and imaging, a physical interpretation of the CRS stacking parameters is available. If the locally constant near-surface velocity $v_{0}$ is known, the CRS formula can be written in a form which allows the interpretation of the stacking parameters as attributes describing two hypothetical emerging wave fronts at the considered surface location $x_{0}$. These are the so-called normal-incidence point (NIP) wave and the normal (N) wave (e.g., Hubral 1983). The NIP wave would be observed at $x_{0}$, if a point-source was placed at the NIP of the zero-offset ray on the imaged reflector in the subsurface, while the $\mathrm{N}$ wave would be obtained if an exploding reflector element - the CRS - was placed around the NIP in the subsurface. The curvature matrix of the NIP wave is $\mathbf{K}_{\text {nip }}$ and the curvature matrix of the $\mathrm{N}$-wave is $\mathbf{K}_{n}$. Because of the link to wavefront curvatures and slopes, these stacking parameters are also called kinematic wavefield attributes. We will use both terms synonymously. The relation of the stacking 
parameters to the kinematic wavefield attributes is given by the following set of equations:

$$
\mathbf{p}_{m}=\frac{1}{v_{0}}(\cos \alpha \sin \beta, \sin \alpha \cos \beta)^{T},
$$

$$
\mathbf{M}_{n i p}=\frac{1}{v_{0}} \mathbf{H K}_{n i p} \mathbf{H}^{T}
$$

$$
\mathbf{M}_{n}=\frac{1}{v_{0}} \mathbf{H K}_{n} \mathbf{H}^{T}
$$

where $\alpha$ is azimuth, $\beta$ is dip angle and $\mathbf{H}$ is the $2 \times 2$ upper left sub matrix of the $3 \times 3$ transformation matrix from the wavefront coordinate system into the registration surface. Since the dip is included into the CRS operator, DMO is automatically accounted for in the stack.

The 3D CRS stack itself is just one product which is obtained by the method. Next to the simulated zero-offset section and volumes containing the optimum kinematic wavefield attributes, the maximum coherence for each $\mathrm{ZO}$ sample is obtained. We will show later, that the coherence may serve as a suitable imaging attribute for hardrock data. After this brief introduction of the 3D CRS method and the kinematic wavefield attributes we discuss now the basic features of the study area.

\section{AREA OF STUDY}

Schneeberg is a town in the state of Saxony, Germany, located close to the border of the Czech Republic (Figure 1). In addition the local surface geology, also in-line and cross-line coordinates of the 3D survey are displayed in Figure 2. These coordinates are used in the 
following to describe the location of vertical sections or specific locations within the 3D volume.

A detailed description of the geology of the survey area is given in (Berger et al. 2011). Here we only describe a few major points which are most relevant for interpretation of the seismic sections. The area is a part of the Saxothuringian fault block system. The structure and tectonics of the area include a massive granitic igneous body overlain by the number of low, medium and high grade metamorphic rock units on the top of the granitic body as shown in Figure 2. There are also patches of sedimentary rocks in the vicinity of this area. The origin of the igneous body is associated with an important CambroOrdovician phase of rifting, which is observed in many regions in Europe (e.g., Emmermann and Wohlenberg 1988). The alternating metamorphic blocks are on top of the igneous body in an inclined position which is typical for areas in the south of the Saxothuringian Zone. This massive igneous body is a prominent structural feature in this area, and strong reflections are expected to originate from the contacts between the metamorphic and igenous rocks between the metamorphic and igneous rocks. For this area of the subsurface high velocities were expected.

As part of the NW-SE striking Finne-Gera-Jachymov fracture system a major steep dipping fault called Roter Kamm runs through the igneous body and is exposed at the surface (Figures 2 and 3). The Roter Kamm strikes with 315-320 NW-SE and shows a dip of 50 to $55^{\circ}$ at the surface. From mining, it is known that the dip increases to about $70^{\circ}$ at a depth of about $500 \mathrm{~m}$. Some conjugate faulting is observed in the SW of the survey area which was assumed to terminate at the Roter Kamm. From previous geological knowledge this area of crossing fault systems was considered to be highly fractured and therefore suitable for geothermal exploration. At the surface the Roter Kamm is characterized by 
fault systems representing a major structure that separates two different types of zones. Prior to the reflection seismic experiment it was expected that the Roter Kamm would form a prominent feature in the seismic cubes. In the next section we describe the application of the CRS method to the Schneeberg data.

[Figure 1 about here.]

[Figure 2 about here.]

[Figure 3 about here.]

\section{COMMON-REFLECTION-SURFACE STACK APPLICATION}

The data have a very poor $\mathrm{S} / \mathrm{N}$ ratio typical for hardrock environments. A very basic sequence of pre-processing was applied to the data, which comprised trace editing, static corrections, top mute, filtering and gain. For the data presented in this paper two-way traveltimes (TWT) from 0 to $500 \mathrm{~ms}$ were left out of the processing and are not displayed. Figure 4a shows the automatic CMP stack for in-line 390. The automatic stack is a part of the procedure to determine CRS attributes (e.g., Mann et al. 1999; Müller 2003) and represents the stack obtained after coherence-based determination of the CMP stacking velocity. It can be described as an automatic way of velocity analysis for each ZO sample. The presented image can not be considered as the optimal result of a conventional CMP processing workflow. The automatic CMP stack displays a poor $\mathrm{S} / \mathrm{N}$ ratio and indicates that a multi-parameter stacking method like 3D CRS involves more traces in the stack than the CMP method might provide an improved image. This was our motivation to apply the data-driven CRS workflow to the Schneeberg hardrock reflection data. 
It is well known that stacks represent an image and are the first step on the way to construct a geological model. It is, however, the only imaging result which is obtained in a purely data-driven fashion, free from any interpretation. In Figure 4b the CRS stack of in-line 390 is shown. Here reflection events are clearer, more continuous and easier to distinguish in comparison to the automatic CMP stack in Figure 4a. Note, that the color amplitude scales are not the same for both plots, since the CRS stack has higher amplitudes and dynamic range than the CMP stack. Many more traces contribute to the CRS image. Although the quality of the CRS-stacked section is already satisfactory because of the increased $\mathrm{S} / \mathrm{N}$ ratio, we also considered the coherence as an additional imaging attribute. As mentioned above, this attribute is obtained in an objective data-driven way during the optimization procedure.

[Figure 4 about here.]

The coherence represents a low-pass positive definite version of the ZO stack image. It should display features very similar to the stack but with a decreased resolution. The coherence image of in-line 390 is shown in Figure 4c. Note again that, the color scale is different from the CMP and CRS sections in this figure, since the coherence is always positive and assumes values between 0 and 1 . For data with a very good $\mathrm{S} / \mathrm{N}$ ratio, the coherence section looks similar to a stacked section where absolute amplitudes are considered. For noisy data, however, the coherence section is the superior choice, since seismic noise does not stack destructively, if absolute amplitudes are used. In the coherence section it is obvious that the steep dipping continuous events crossing the whole section are of similar relevance as the events in the top left corner. This conclusion is not so easily derived from the CRSstacked section (Figure 4b). Therefore, we concluded that the coherence provides a suitable 
imaging attribute for the structural interpretation of this particular set of hardrock data. The coherence value directly reflects the physical relevance of the stacked event and may therefore be used as a weight to further decrease the noise level in the CRS-stacked section.

This weighting is performed in Figure 4d by simply multiplying the stacked amplitude with the respective local coherence. The $\mathrm{S} / \mathrm{N}$ ratio of the weighted stack is considerably improved when compared with the unweighted CRS stack (Figure 4b). The two prominent events present in this section are related to the reflections from the top and bottom of clayey schists which are present in the SE of the survey area (see geological map in Figure 2). The strong events in the top left are located in the permian sediments above the granitic intrusion. This area was subject of intensive uranium mining (e.g., Berger et al. 2011). Since the emphasize in this paper is the application of the CRS method to hardrock data and the establishment of a data-driven workflow using various imaging attributes, no detailed interpretation of the images is given here. For this purpose we refer to (Lüschen et al. 2015).

In Figure 5 we present the coherence section of in-line 369. The coherence section show many crossing events typical for hardrock data. Several NE-SW dipping structures are dominating the left part of the section. Between cross-line coordinates 200 and 500 we observe strong events for two-way-times larger than $2 \mathrm{~s}$, which are not present elsewhere. This structure was called Schneeberg body. The Schneeberg body is a geological feature which represents the scattered energy below Roter Kamm at the position of xline 200 near $2 \mathrm{~s}$ time. On this in-line no obvious evidence of the Roter Kamm can be detected. The surface location of the Roter Kamm is at about cross-line coordinate 230 for this in-line section. Events attributable to Roter Kamm were actually only obtained on a few in-line sections. The seismic appearance of the Roter Kamm is characterized by a considerably smaller coherence when compared with the coherence of the conjugate faults. Conjugate 
faults are visible in the Figure 5 and 3D cube. On most in-lines the presence of the Roter Kamm in the study area is not expressed directly by a seismic event but by the fault throw of the conjugated faults or increased attenuation (Lüschen et al. 2015). In contrast to the geological model derived prior to the seismic experiment, the conjugate faults do not terminate at the Roter Kamm (Figure 3) but penetrate through it and extend considerably further SW.

[Figure 5 about here.]

A 3D view of coherence along in-line 369, cross-line 650 and the time-slice at $2300 \mathrm{~ms}$ TWT is shown in Figure 6. The features already described for in-line 369 in Figure 5 are recognized in this visualization as well, mainly the conjugate faults and the top of the Schneeberg body. The cut at cross-line 650 is shown in Figure 6. The reflections from top and bottom of the clayey schist are dominating. The time slice hides a large part of the Schneeberg body visible on the in-line section, we can, however, observe that some strong events of this structure are present at about $3000 \mathrm{~ms}$. A further feature can be detected in the time-slice. It is a fault system in the NE corner of the slice which cuts into the Schneeberg body. From the visual inspection of the various seismic images we can conclude that the study area comprises a huge inventory of faults with a concentration in the central south-western part. These faults might provide a suitable fracture system for geothermal exploitation.

[Figure 6 about here.] 


\section{CONCLUSION}

We have presented the application of a 3D CRS-based workflow to the Schneeberg 3D hardrock reflection seismic data. The $\mathrm{S} / \mathrm{N}$ ratio of these data is very low and the initial automatic CMP stack indicated that multi-parameter processing methods, which use more traces in the stack might improve the image quality. The data are dominated by diffractions generated at various steep dipping fracture zones. Steep structures and a huge number of diffraction tails interfere or lead to a complicated pattern of conflicting dips. The CRS stack showed a considerably improved $\mathrm{S} / \mathrm{N}$ ratio and provided a reasonable basis for geological interpretation. However, plots of the coherence actually delivered good images for interpretation for this particular type of hardrock data. Despite the reduced resolution compared to the stack, we consider the coherence as a suitable imaging attribute, since it is an objective measure of the local similarity in the wavefield and directly reflects the physical relevance of the corresponding seismic event. For the considered data the coherence is a data-driven imaging attribute particularly helpful for the interpretation of hardrock data. Weighting, i. e., multiplying the stacks with the coherence further improved the $\mathrm{S} / \mathrm{N}$ ratio of the resulting sections and supported the interpretation of the images. Similar conclusions apply to the display of the coherence-weighted time slices, which provided the best images.

The reflections from top and bottom of the clayey schists represent the most prominent features in the data. We could also nicely map a sequence of conjugate faults. Their lateral extent at depth seems to be small which does not coincide with the surface expression of these faults. Prior to this experiment the Roter Kamm was expected to be the most

prominent structure in the data. We found, however, that there are only faint hints of this fault in the data and the respective coherence is much smaller than the coherence of 
the sequence of conjugate faults. Another prominent feature of the data was detected in the SW of the survey area. This feature represents a region with a very high number of aligned events below $1.5 \mathrm{~s}$ TWT. This area of high coherence is located in a region where several fracture systems intersect and may have a high fracture density. Whether these fractures are open or mineralized cannot be decided from the current state of processing. Open fractures would make this area a favorable region for a geothermal heat exchanger. It is known, however, that many fracture systems in the North of the measurement surface are mineralized, which initiated the mining activities in this area.

\section{OUTLOOK}

All options to improve the signal quality of the pre-stack seismic data will help to better image the subsurface of hardrock systems. Pre-stack time and depth migration of hardrock data will rely on the respective velocity model input. Because of the poor data quality, the estimation of CRS attributes is not necessarily stable and all processes relying on these attributes may be compromised. This includes the determination of time migration velocities. Key element in the successful processing of hardrock data is the enhancement of the pre-stack data quality. Partial CRS stacks (e.g., Baykulov and Gajewski 2009) might be an option to improve the data quality in the pre-stack domain. Seismic data from a hardrock subsurface are largely dominated by diffractions, which are not optimally fitted by the CRS operator. Processing the data with the i-CRS operator (e.g., Schwarz et al. 2014) might improve the determination of wave field attributes, since it better fits diffractions. High quality attributes will result in more reliable time migration velocities, improved NIP wave tomography and better pre-stack data enhancement opportunities. Another option of prestack data improvement is given by the partial time migration introduced by (Dell et al. 
2012). This tool also has a pre-stack data enhancement facility and requires reasonable time migration velocities to be applied effectively.

\section{ACKNOWLEDGMENTS}

Special thanks go to the BMWi (Bundesministerium für Wirtschaft und Energie), the Federal Ministry for Economic Affairs and Energy (Project 0325363C) for funding the field work and the processing. The discussions with the project partners of the Leibniz Institute for Applied Geophysics (LIAG), Hannover, Germany, and TU Bergakademie Freiberg, Freiberg, Germany, are highly appreciated. We would like to thank the Applied Geophysics Group Hamburg for continuous discussions. This work was also partially supported by the sponsors of the Wave Inversion Technology (WIT) Consortium. Dela Spickermann, Michael Böttinger and Hendryk Bockelmann from DKRZ (Deutsches Klimarechenzentrum) greatly helped to meet computational demands. Detailed comments by the reviewers and the associate editor helped to improve the original version of the manuscript. 


\section{REFERENCES}

Baykulov M., Dümmong S. and Gajewski D. 2011. From time to depth with CRS attributes. Geophysics 76, S151-S155.

Baykulov M. and Gajewski D. 2009. Prestack seismic data enhancement with partial common-reflection-surface (CRS) stack. Geophysics 74, V49-V58.

Berger H.-J., Felix M., Görne S., Koch E., Krentz O., Förster A., Förster H.-J., Konietzky H., Lunow C., Walter K., Schütz H., Stanek K. and Wagner S. 2011. Tiefengeothermie Sachsen. Landesamt für Umwelt, Landwirtschaft und Geologie. Schriftenreihe, No. 9.

Bergler S., Hubral P., Marchetti P., Cristini A. and Cardone G. 2002. 3D common-reflectionsurface stack and kinematic wavefield attributes. The Leading Edge 21, 1010-1015.

Dell S. and Gajewski D. 2011. Common-reflection-surface-based workflow for diffraction imaging. Geophysics 76, S187-S195.

Dell S., Gajewski D. and Vanelle C. 2012. Prestack time migration by common-migratedreflector-element stacking. Geophysics $\mathbf{7 7}$, S73-S82.

Dümmong S. and Gajewski D. 2008. A multiple suppression method via CRS attributes. 2008 SEG Expanded Abstracts 2531-2535.

Duveneck E. 2004. Velocity model estimation with data-derived wavefront attributes. Geophysics 69, 265-274.

Emmermann R. and Wohlenberg J. 1988. German Continental Deep Drilling Program (KTB). Springer.

Hertweck T., Schleicher J. and Mann J. 2007. Data stacking beyond CMP. The Leading Edge 26, 818-827.

Hubral P. 1983. Computing true amplitude reflections in a laterally inhomogeneous earth. Geophysics 48, 1051-1062. 
Lüschen E., Görne S., von. Hartmann H., Thomas R. and Schulz R. 2015 3D Seismic Survey for Geothermal Exploration in Crystalline Rocks in Saxony, Germany: Geophysical Prospecting (this issue).

Malehmir A., Durrheim R., Bellefleur G., Urosevic M., Juhlin C., White D. J., Milkereit B. and Campbell G. 2012. Seismic methods in mineral exploration and mine planning: A general overview of past and present case histories and a look into the future. Geophysics 77, WC173-WC190.

Mann J., Jäger R., Müller T. and Hubral P. 1999. Common-reflection-surface-stack - a real data example. Journal for Applied Geophysics 42, 283-300.

Milkereit B., Berrer E. K., King A. R., Watts A. H., Roberts B., Adam E., Eaton D. W., Wu J. and Salisbury M. H. 2000. Development of 3-D seismic exploration technology for deep nickel-copper deposits-A case history from the Sudbury basin, Canada. Geophysics 65, 1890-1899.

Müller N. A. 2003 The 3D Common-Reflection-Surface stack - Theory and application: Master's thesis University of Karlsruhe.

Schwarz B., Vanelle C., Gajewski D. and Kashtan B. 2014. Curvatures and inhomogeneities: An improved common-reflection-surface approach. Geophysics 79, S231-S240. 


\section{LIST OF FIGURES}

1 Geographical map of Germany with the study area marked. . . . . . . . . . 19

2 Geological map of the study area with in-line/cross-line coordinates. The white line indicates the location of the geological profile in Figure 3. . . . . 20

3 Geological cross section of the study area following approximately the white line in Figure 2, modified after Berger et al. (2011) . . . . . . . . . . . . . .

4 (a) Automatic CMP Stack, (b) CRS stack, (c) coherence, (d) coherence weighted stack of in-line $390 . \ldots \ldots 22$

$5 \quad$ Coherence section of in-line $369 . \ldots \ldots \ldots$

$63 \mathrm{D}$ view of coherence for in-line 369, cross-line 650 and the time slice at 2300 ms TWT. The arrow at the left bottom indicates North. . . . . . . . . . . . 24 


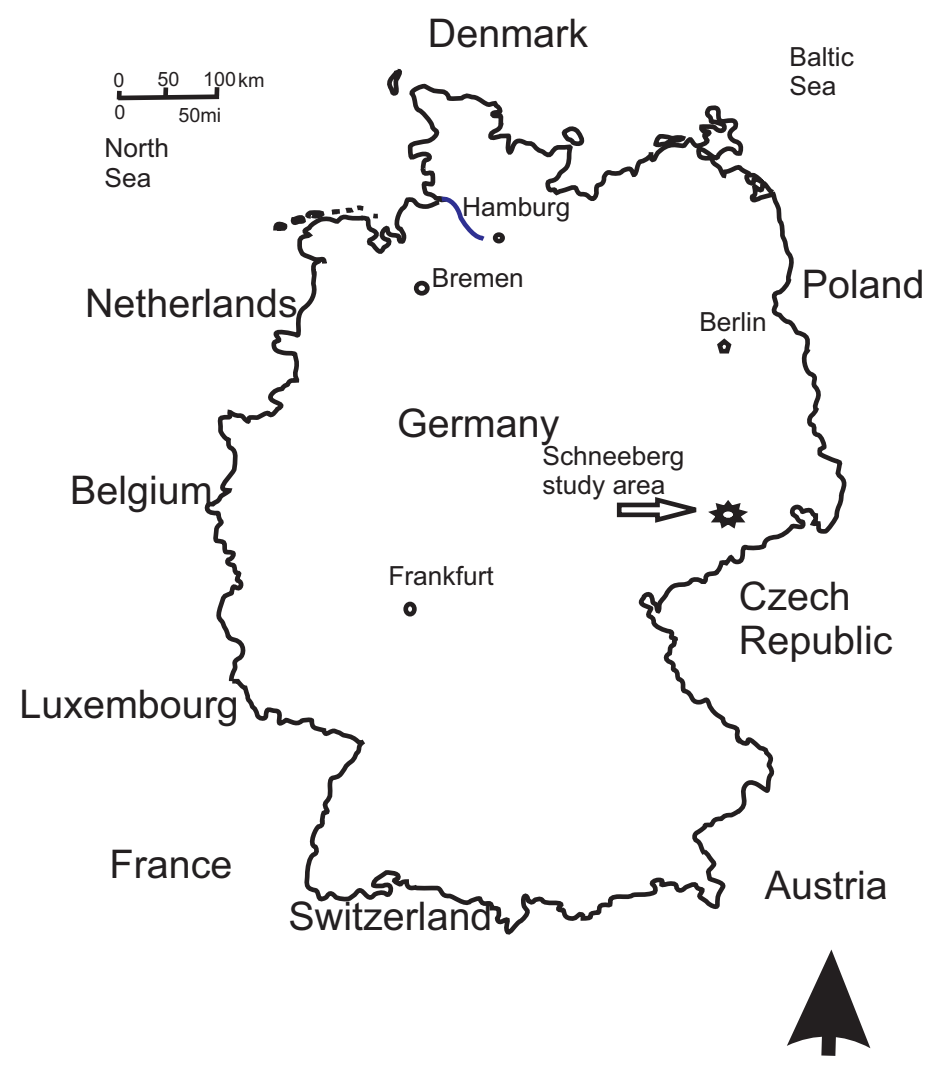

Figure 1: Geographical map of Germany with the study area marked. 


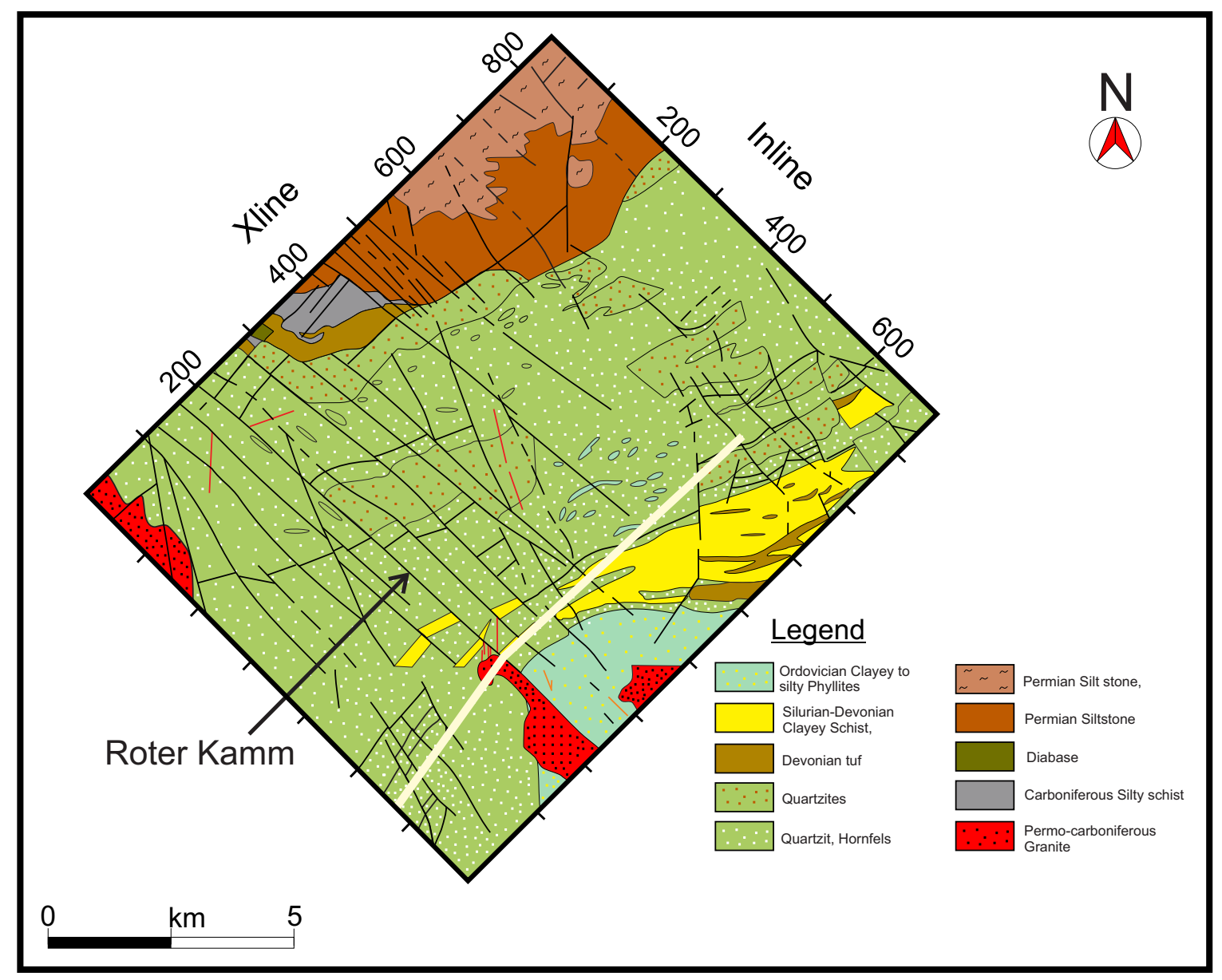

Figure 2: Geological map of the study area with in-line/cross-line coordinates. The white line indicates the location of the geological profile in Figure 3. 


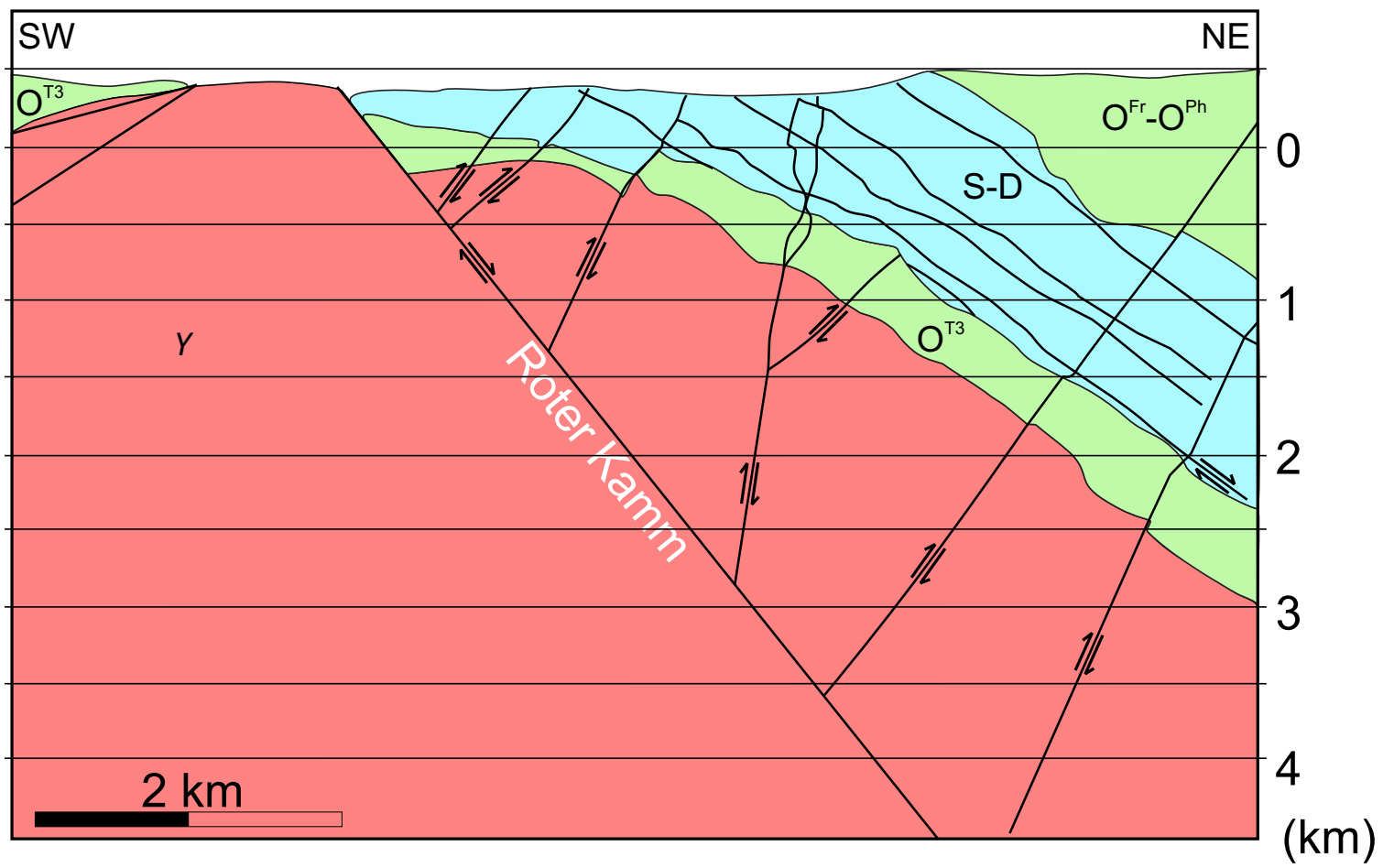

\section{LEGEND}

\section{$\begin{array}{llll}\mathrm{S}-\mathrm{D} & \begin{array}{l}\text { Ordovician Silurian } \\ \text { Devonian rock }\end{array} & \mathrm{O}^{\top 3} & \begin{array}{l}\text { Ordovician Phyllites } \\ \text { Diabase Quartzite }\end{array}\end{array}$ \\ $\mathrm{O}^{\mathrm{Fr}}-\mathrm{O}^{\mathrm{Ph}}$ Ordovician Phyllites Quartzite \\ Older granite with eruptive complex and mirror stone like rock}

Faults

Figure 3: Geological cross section of the study area following approximately the white line in Figure 2, modified after Berger et al. (2011). 


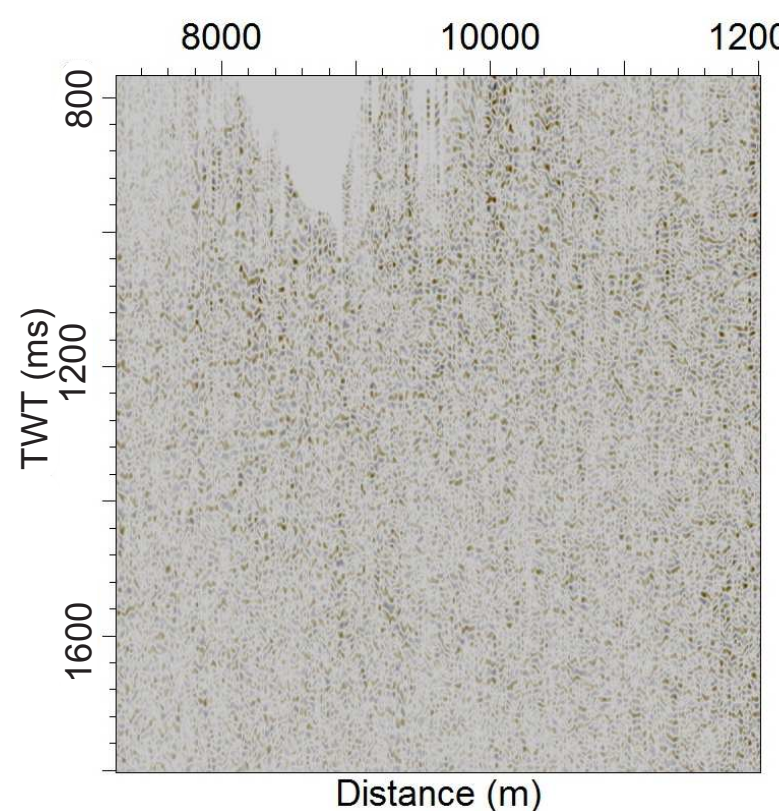

(a)

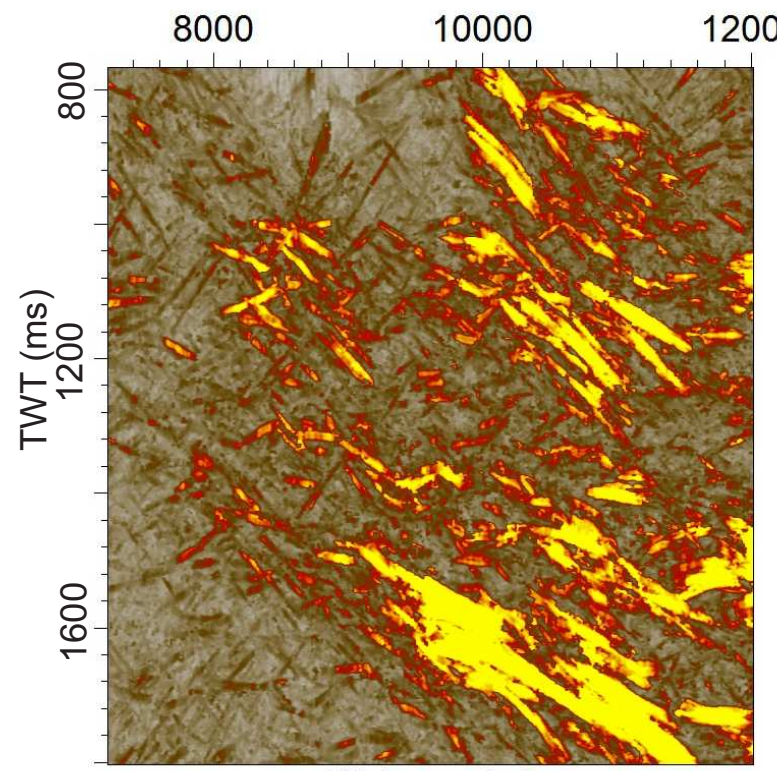

Distance (m)

(c)

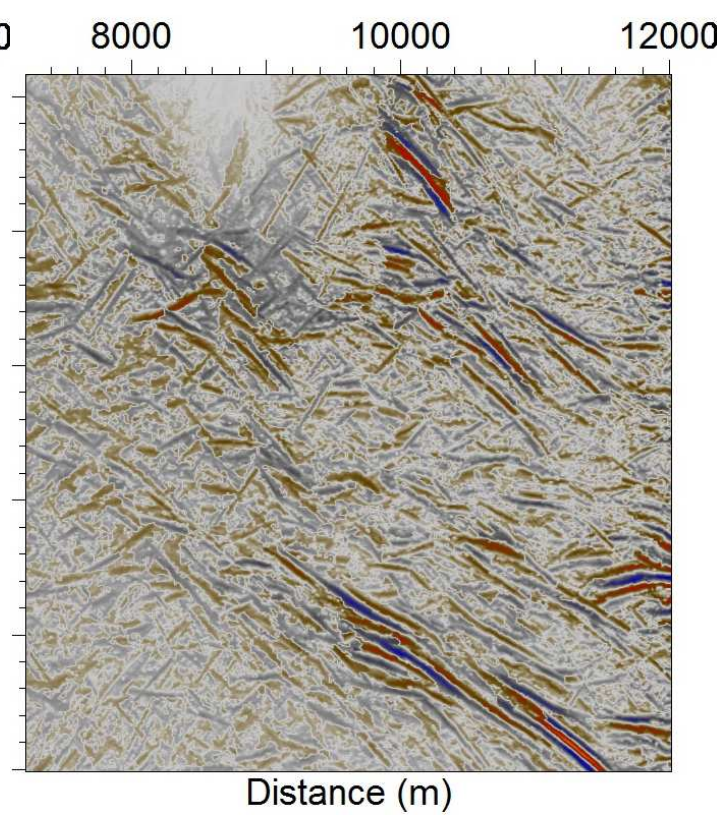

(b)

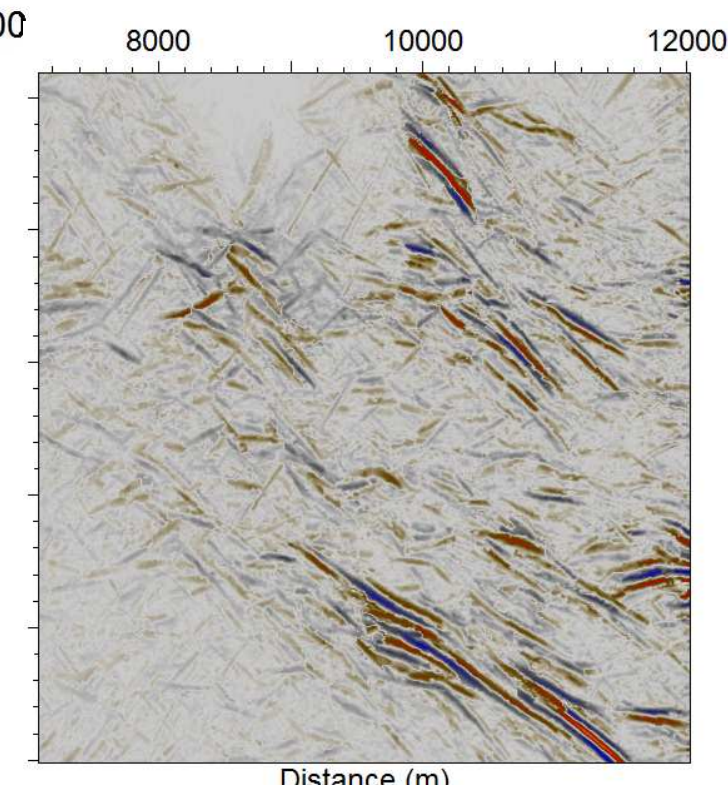

(d)

Figure 4: (a) Automatic CMP Stack, (b) CRS stack, (c) coherence, (d) coherence weighted stack of in-line 390. 


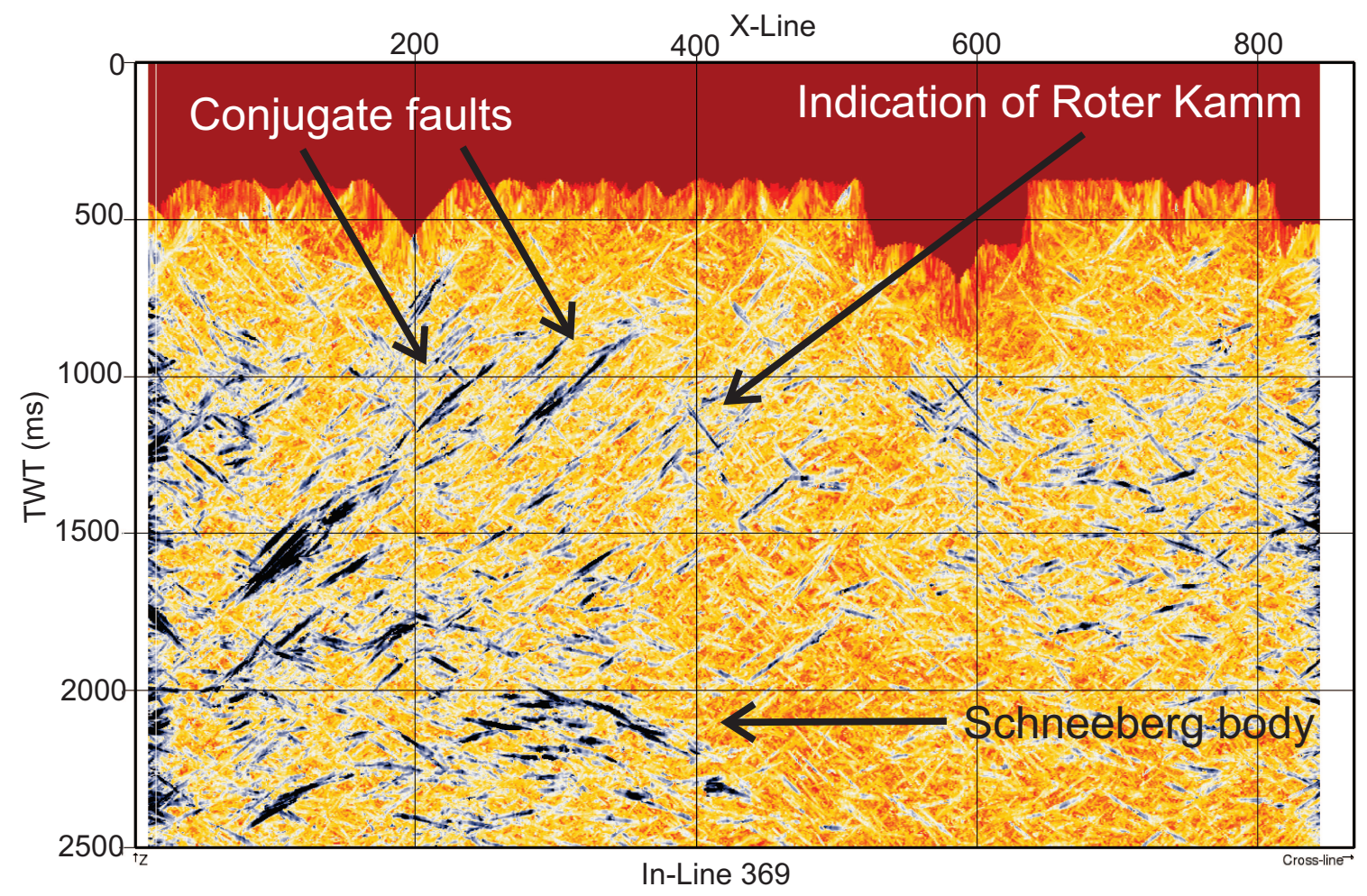

Figure 5: Coherence section of in-line 369. 


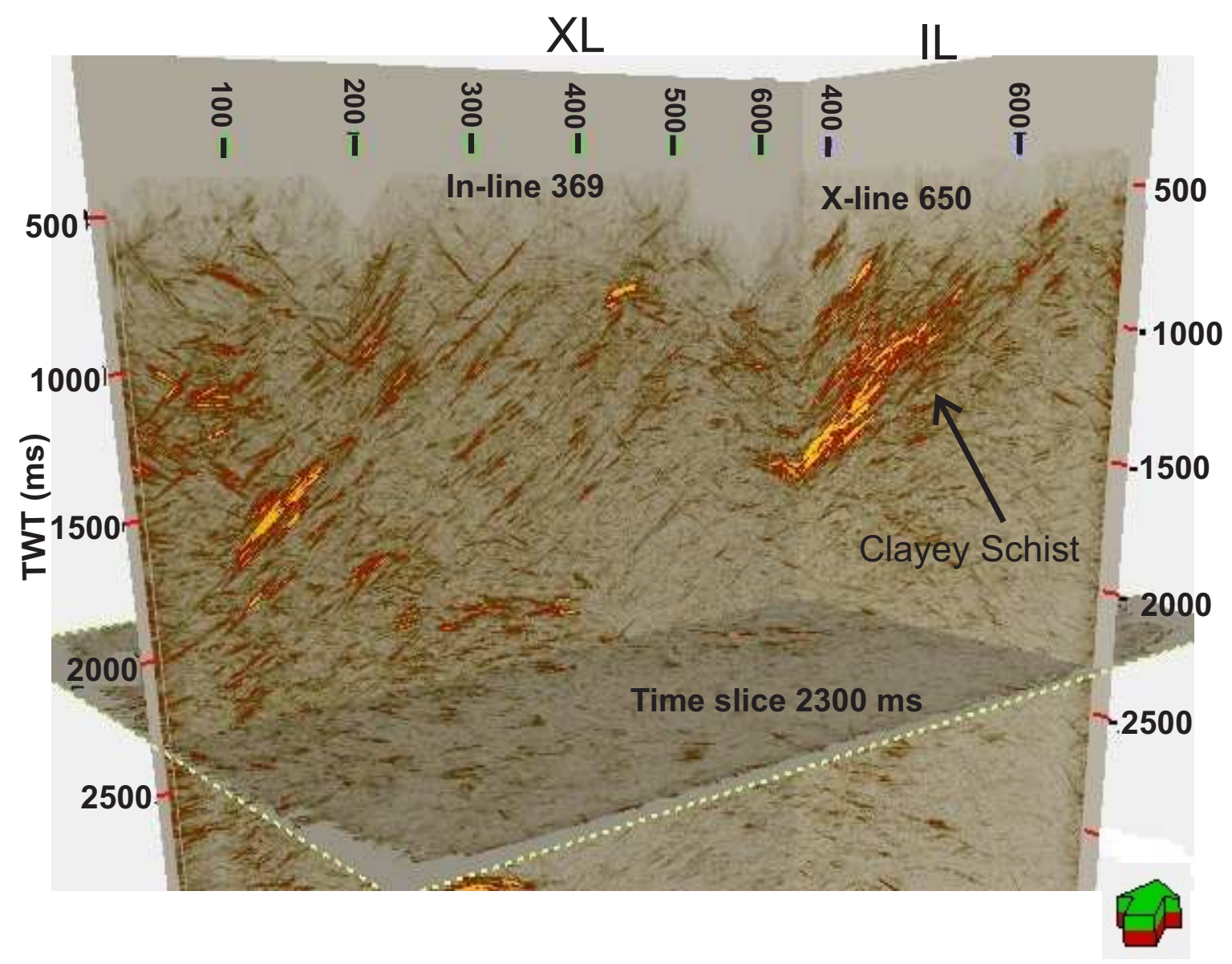

Figure 6: 3D view of coherence for in-line 369, cross-line 650 and the time slice at $2300 \mathrm{~ms}$ TWT. The arrow at the left bottom indicates North. 